\title{
Management of Recurrent Enterocolitis Following Soave's Pull-Through in a Child With Hirschsprung's Disease: Value of Botox Injections
}

\author{
Kashif Chauhan ${ }^{\mathrm{a}, \mathrm{b}}$, Gulwish Moghul ${ }^{\mathrm{a}}$, Shailinder Singh ${ }^{\mathrm{a}}$
}

\begin{abstract}
Recurrent enterocolitis (RE) is a common problem following Soave's pull-through for Hirschsprung's disease. The main cause of this is outlet obstruction. This can be anatomical (stricture, stenosis, and twist of bowel) or histological (aganglionic or transition zone pull through). Outlet obstruction leading to RE can be from two factors that are specific to Soave's pull through: hypertonicity of the internal anal sphincter or residual cuff. A residual cuff can be managed through excision of the cuff, although this is a major surgical undertaking. We report a successful use of Botox in a child with RE secondary to residual cuff following primary laparoscopic Soave's operation. A Botox injection was administered under general anesthesia at five separate sittings. This case demonstrates that repeated Botox injections can be a valuable alternative to colostomy, excision of residual cuff or a redo pull-through when a cuff is thought to be responsible for RE.
\end{abstract}

Keywords: Hirschsprung's disease; Soave pull-through; Enterocolitis; Anal sphincter; Botox

\section{Introduction}

Most children with Hirschsprung's disease remain well after Soave's pull-through surgery, but some continue to have persistent obstructive symptoms. This can present in the form of constipation, recurrent attacks of abdominal distension (requiring insertion of a flatus tube or rectal washouts) or recurrent attack of enterocolitis which often leads to surgical intervention. These interventions depend on the presumed underlying cause. Various causes responsible for recurrent enterocolitis (RE) are: 1) stricture or stenosis of the anal canal following a pull-through operation; 2) aganglionic pullthrough bowel; 3) pull-through of the transition zone; 4) a

\footnotetext{
Manuscript accepted for publication December 15, 2016

aNottingham University Hospital, Queen's Medical Centre, UK

${ }^{b}$ Corresponding Author: Kashif Chauhan, Nottingham University Hospital, Queen's Medical Centre, UK. Email: mkashifdr@yahoo.com
}

doi: https://doi.org/10.14740/jmc2720w motility disorder of the bowel above the pull-though area, which some believe to be secondary to neuronal intestinal dysplasia; and 5) problems particular to the anal canal in Hirschsprung's disease [1]. Problems particular to the anal canal are only considered once the other main causes have been ruled out.

Problems particular to the anal canal can be due to internal sphincter hypertonicity with a non-relaxing sphincter [1] or from a cuff around the pull-through bowel [2]. There are several studies on the role of Botox in the treatment of internal sphincter hypertonicity, including the use of multiple injections up to a maximum of four times [3]. The group of surgeons who believe that the episodes of RE are secondary to an abnormal cuff advocate the excision of the cuff, which is a major surgical undertaking. However, there are currently no studies published on the use of Botox in patients with an abnormal cuff. We report the successful use of five injections of Botox in a patient who presented with recurrent attacks of enterocolitis following a laparoscopic-assisted Soaves' primary pull-through procedure, where the recurrent attacks were secondary to an abnormal cuff.

\section{Case Report}

This case is a full-term healthy neonate with a birth weight of $3.8 \mathrm{~kg}$. On the second day of life, she developed bilious vomiting and abdominal distension with no passage of meconium. An upper and lower gastrointestinal (GI) contrast study was performed. The upper GI contrast study was normal, but the lower contrast study showed a dilated gas filled distal bowel in the region of the descending colon. Thus, a rectal suction biopsy was done on the third day of life, with histology confirming Hirschsprung's disease with the absence of ganglionic cells. Once diagnosed, she was kept as an inpatient for a few days, whilst she was started on a twice daily rectal washout regimen. She was discharged on the same regimen, with a date scheduled for a planned pull-through procedure.

At 10 weeks old, a laparoscopic-assisted Soaves' pullthrough procedure was performed. The cuff was divided but not excised, with removal of $4-5 \mathrm{~cm}$ of the aganglionic segment at the recto-sigmoidal region. She recovered well and was discharged home on the fourth postoperative day. However, she was readmitted on day 5 postoperatively with pyrexia, feeling generally unwell, lethargy, poor feeding, vomiting and abdom- 


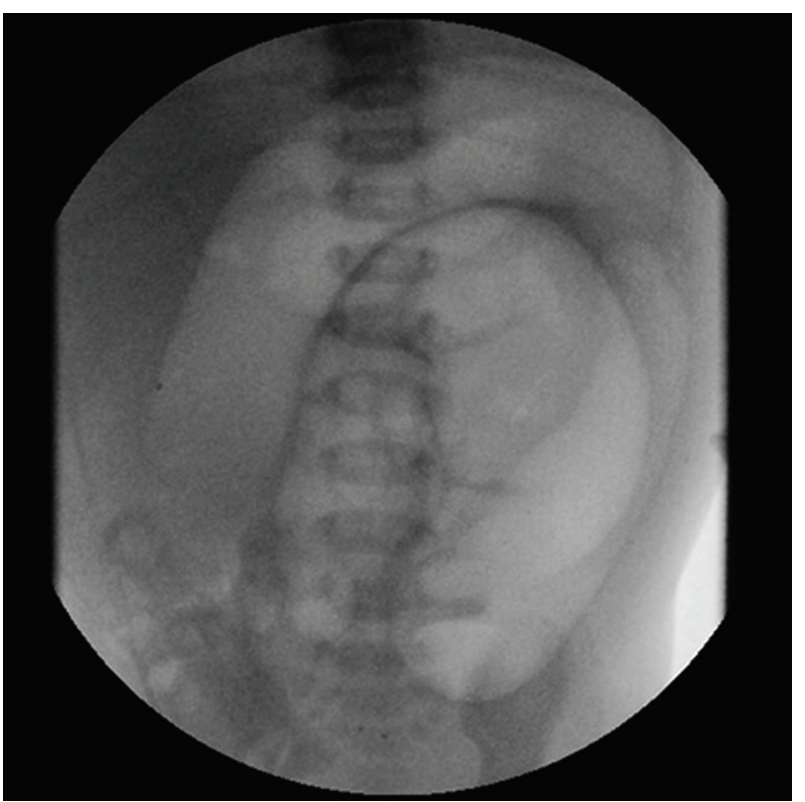

Figure 1. First episode of enterocolitis 5 days after laparoscopic Soave's pull-through.

inal distension (Fig. 1). She was diagnosed with Hirschsprung's enterocolitis. Rectal examination was performed, which was consistent with a rolled up cuff. It was thought that the split, but un-excised cuff had joined together, rolling up at ano-rectal junction causing obstruction. This would have then led to enterocolitis. According to our standard practice, we usually divide the cuff and excise it completely. However, in this case we divided the cuff but did not excise it. She then had colonic decompression with a rectal catheter along with further rectal washouts and intravenous antibiotics for a total of 5 days. She was discharged home on day 5 of admission.

Once discharged, she continued to experience abdominal distension. Thus, she was brought back in 6 weeks postoperatively for an examination under anesthesia (EUA) of the anal region, with a repeat rectal biopsy and a Botox injection. The EUA did not reveal any stricture. She was discharged home later that day with laxatives. The repeat biopsy showed that the pull-through region contained ganglionic cells with no evidence of an aganglionic region.

She remained well for a few weeks following her first Botox injection. However, she returned to A\&E 3 weeks after the initial injection with another episode of enterocolitis. She was managed with intravenous antibiotics and repeated rectal washouts, including a second Botox injection. This was administered 4 weeks after the first injection. She recovered well and was sent home with oral metronidazole as a prophylaxis. Despite this, she continued to develop recurrent episodes of enterocolitis requiring repeated hospital admissions. Following an extensive discussion with our surgical colleagues, it was decided that a third Botox injection should be administered. This was performed 3 months after the second injection, improving her short-term outcome, but failing to prevent further episodes of enterocolitis leading to several hospital admissions.

A fourth Botox injection was administered 7 months after the third one, with a repeat rectal biopsy performed at the same time. This, again, showed a ganglionic pull-through segment. However, she continued to present with episodes of enterocolitis. In between these episodes, she was reviewed regularly, both in the outpatients department and as a day attender on the ward. Her stool pattern ranged from normal to watery in consistency with the use of laxatives. Further detailed discussion led to the decision to administer a fifth Botox injection. This was given 4 months after the fourth one.

Close monitoring revealed that after the fifth injection of Botox, she no longer developed enterocolitis. She was reviewed in the outpatient department at 3,6, 9, 12 and 18 months after the fifth Botox injection with not a single episode of enterocolitis. In fact, her bowel habit returned to normal with a reasonable consistency without the need for laxatives.

This case was very difficult to manage with repeated episodes of enterocolitis requiring hospital admissions and intravenous antibiotics. Over the course of 2 years, this patient was administered Botox five times, with no further recurrence after the last injection at age 2. She is now 5 years of age and has been symptom-free for the last 3 years. She is growing as expected, achieving the developmental milestones appropriate for her age.

\section{Discussion}

Patients with Hirschsprung's disease treated with a pullthrough operation can present with postoperative obstructive symptoms leading to RE. The obstructive pathology leading to RE can be caused by four main factors: 1) anatomical or mechanical factors (anastomotic strictures, anal stenosis or kinks in the bowel) [4]; 2) histological factors (a retained aganglionic segment or transition zone pull-through); 3) motility issues (secondary to neuronal intestinal dysplasia); and 4) two disorders particular to a Soave pull-through, i.e. internal sphincter hypertonicity and an abnormal (retained) cuff at the level of the anal canal.

The main anatomical causes of obstruction are as follows: 1) stenosis at the level of the anastomosis; 2) a stricture of the anal canal; or 3) a kink or twist of the pull-through bowel. Anal stenosis is diagnosed by a per-rectal examination and can usually be managed with dilatation; a secondary surgical procedure is rarely necessary. Strictures within the pull-through bowel have also been described and are probably secondary to ischemic damage. These can, again, be diagnosed by a perrectal examination and may require surgical correction. In the case described, anal stenosis and strictures were ruled out through a per-rectal examination. A kink or a twist of the pullthrough bowel is diagnosed by a contrast enema study. However, in the case described, the entire pull-through procedure was performed laparoscopically, thus observed in real-time, so we were confident that there had not been a kink or twist of the pull-through bowel. Hence, a contrast study was not required to rule out these two possibilities.

The main histological causes of obstruction are as follows: 1) aganglionic bowel at the level of the pull-through or 2 ) the transition zone remaining at the level of pull-through. 


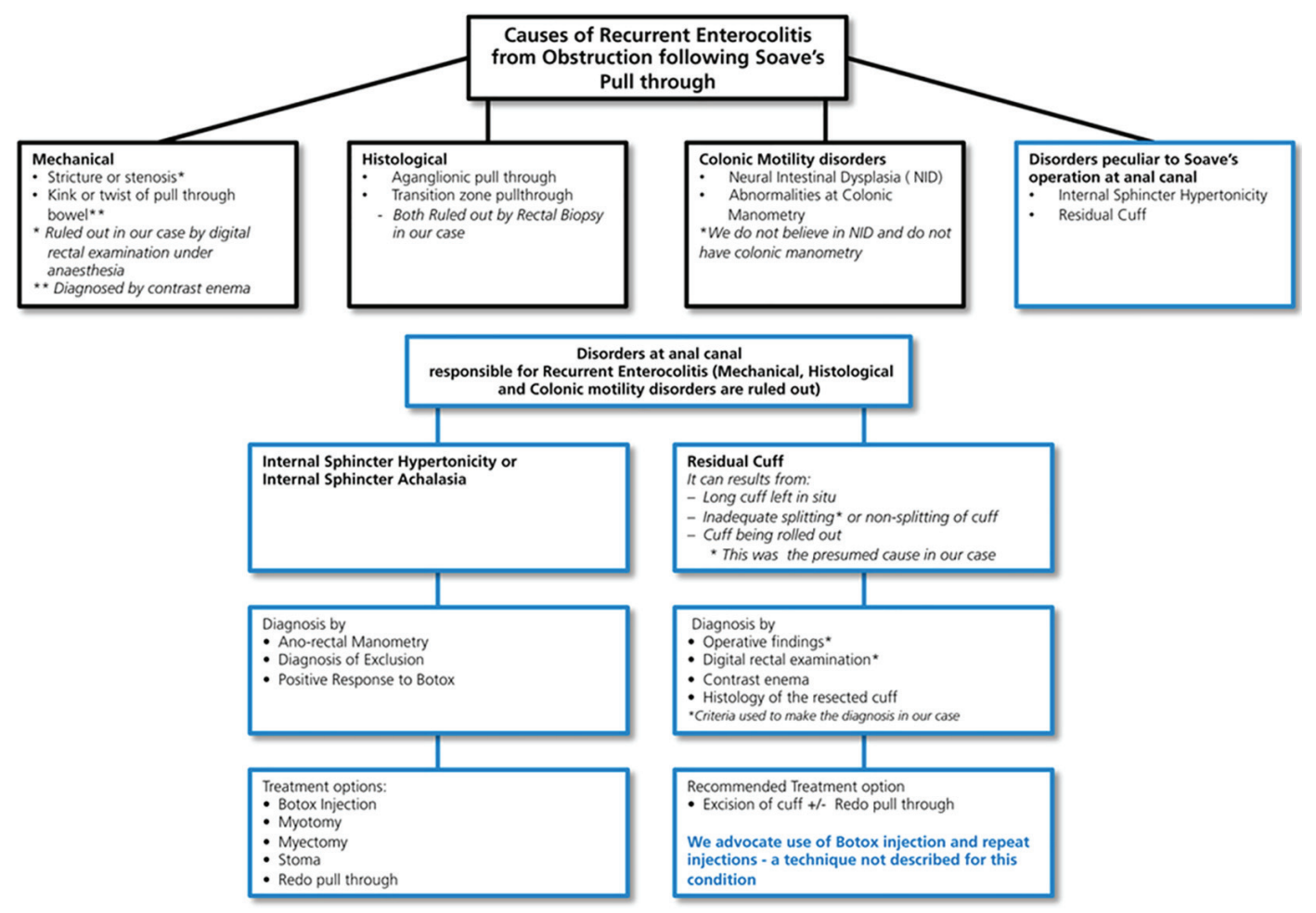

Figure 2. Treatment algorithm with RE and residual cuff after Soave's pull-through operation.

These are usually due to histological misdiagnosis. However, the aganglionosis may also be acquired after a successful initial operation. This has been described in the literature, but is debatable. Since we are aware of the histological pitfalls at the time of pull-through for Hirschsprung's disease, our standard practice is to confirm the presence of ganglion cells by frozen section before the pull-through. We also send a donut (full thickness circumferential tissue of intestinal wall) at the level of pull-through. We await histological confirmation of ganglion cells in the entire circumference of the donut before waking the patient up from general anesthesia. Through this practice of dual histological confirmation intra-operatively, we have been successful in preventing aganglionic and transition zone pull-through in our unit. In this case, we performed a rectal biopsy, which did not reveal histological evidence of aganglionic bowel or a transition zone pull-through. In fact, a rectal biopsy was performed twice to rule out the possibility of acquired aganglionosis.

We are aware of the viewpoint that motility issues can be one of the causes of RE. These are diagnosed by colonic manometry. This test is not available in many centers, with the technique still in the evolution phase. Standard parameters have also yet to be agreed upon. Unfortunately, we do not have the facility for colonic manometry at our center. Motility issues are thought to be due to neuronal intestinal dysplasia by some authors. However, this is a debatable diagnosis based on specific criteria [3]. Our pathology department does not agree with this diagnosis or criteria, hence we were unable to make this diagnosis.

Once the anatomical, histological and motility issues are ruled out, one is left with the possibility of internal sphincter hypertonicity or a retained (abnormal) cuff as the cause of RE [5]. Anorectal manometry (ARM) is required to make the diagnosis [4]. This is performed under intravenous ketamine, as the results are inaccurate under general anesthesia. In our setup, ARM is performed in the operating theater as a day case procedure. It is, thus, an invasive investigation. In addition, ARM cannot distinguish between cuff problems and hypertonicity. Hence, we did not perform ARM in this case. Moreover, we were confident of the diagnosis being due to cuff issues rather than hypertonicity of the sphincter.

We were confident of the diagnosis of a cuff-related issue for two reasons. Firstly, we did not excise the cuff but had only divided it. Divided and un-excised cuffs are known to roll up and cause obstruction. Secondly, during the rectal examination, tightness could be felt at the upper end of the anal canal (from a rolled up cuff) rather than uniform tightness in the entire anal canal (high sphincter pressure).

Contrast examination is an invasive procedure, involves radiation, and may not discriminate between tightening of the entire anal canal (as in high sphincter pressure) and tightening at the upper end of anal canal (rolled up cuff). Hence, we did not perform a contrast enema.

Our standard practice involves dividing the cuff and excising it completely. However, in this case the cuff was divided but not excised. On digital per-rectal examination, the little finger could be introduced with ease. The rolled up cuff could also be felt above the suture line of the anastomosis. We are aware that high sphincter pressures can cause obstructive symptoms. However, this would have been demonstrated by a 
uniform tightness along the entire anal canal rather than a ringlike tightening at the upper end of anal canal. Thus, the rectal examination performed on postoperative day 5 was consistent with the diagnosis of a rolled up cuff. We hypothesised that the split cuff had joined together and had rolled up at the analrectal junction causing obstruction leading to RE.

The recommended treatment for an abnormal cuff is simply to excise the cuff [6]. However, this is a major surgical undertaking. There are a plethora of literature on the role of Botox injections in the treatment of internal sphincter hypertonicity, including the use of multiple injections (up to a maximum of four times) [7]. We have administered multiple injections of Botox for the treatment of hypertonicity too. However, there have not been studies published on the use of Botox in patients with an abnormal cuff. We report the successful use of five injections of Botox in a patient who presented with RE following a laparoscopic-assisted Soave's primary pull-through, where the recurrent attacks were secondary to an abnormal cuff. It is important to note that we do not know the mechanism of action of these injections. We believe that the Botox injections were a temporary measure in delaying attacks whilst the obstructive symptoms improved with time.

Botox is a Clostridium botulinum type A toxin. It can effectively weaken a muscle for a period of 3 - 4 months. Botulinum injections act on the parasympathetic nerve terminals of the neuromuscular junction in striated muscle by blocking acetylcholine release. It also acts on non-adrenaline mediated neural output in smooth muscles, causing transient paralysis of the muscle fiber. It reduces the anal resting pressure, an effect that persists for 2 - 3 months [8]. A study by Langer reported that 14 patients with internal sphincter achalasia causing bowel obstruction saw significant improvement in their symptoms following treatment with the botulinum toxin injection (BTI). Out of the 14, four had prolonged improvement after one injection, while nine continued to have recurrent symptoms, requiring one to four injections [1]. It was difficult to predict which patients would profit from BTI [9]. Frequent injections are necessary. Our case report suggests that a retained cuff can be successfully treated with repeated Botox injection as shown in the treatment of non-relaxing internal anal sphincter (Fig. 2).

\section{Conclusion}

Repeated intra-sphincteric BTIs is a safe and less invasive alternative to cuff excision for the treatment of RE following Soave's pull-through. This is when the cause of RE is as a result of obstruction at the anal canal due to abnormality of the cuff.

Cuff problems after Soave's pull-through procedure respond very well with repeated Botox injection.

Major re-do surgery is avoided with the use of BTIs.

\section{Conflicts of Interest}

None.

\section{Author Contributions}

Kashif Chauhan collected all the data, searched and reviewed the published articles and wrote the paper. Gulwish Moghul helped in writing the paper. Shailinder Singh supervised consultant, checked the paper and wrote the treatment.

\section{References}

1. Langer JC. Persistent obstructive symptoms after surgery for Hirschsprung's disease: development of a diagnostic and therapeutic algorithm. J Pediatr Surg. 2004;39(10):1458-1462.

2. Han-Geurts IJ, Hendrix VC, de Blaauw I, Wijnen MH, van Heurn EL. Outcome after anal intrasphincteric Botox injection in children with surgically treated Hirschsprung disease. J Pediatr Gastroenterol Nutr. 2014;59(5):604607.

3. Frykman PK, Short SS. Hirschsprung-associated enterocolitis: prevention and therapy. Semin Pediatr Surg. 2012;21(4):328-335.

4. Dickie BH, Webb KM, Eradi B, Levitt MA. The problematic Soave cuff in Hirschsprung disease: manifestations and treatment. J Pediatr Surg. 2014;49(1):77-80; discussion 80-71.

5. Levitt MA, Dickie B, Pena A. Evaluation and treatment of the patient with Hirschsprung disease who is not doing well after a pull-through procedure. Semin Pediatr Surg. 2010;19(2):146-153.

6. Temple SJ, Shawyer A, Langer JC. Is daily dilatation by parents necessary after surgery for Hirschsprung disease and anorectal malformations? J Pediatr Surg. 2012;47(1):209-212.

7. Marty TL, Seo T, Sullivan JJ, Matlak ME, Black RE, Johnson DG. Rectal irrigations for the prevention of postoperative enterocolitis in Hirschsprung's disease. J Pediatr Surg. 1995;30(5):652-654.

8. Chumpitazi BP, Fishman SJ, Nurko S. Long-term clinical outcome after botulinum toxin injection in children with nonrelaxing internal anal sphincter. Am J Gastroenterol. 2009;104(4):976-983.

9. Koivusalo AI, Pakarinen MP, Rintala RJ. Botox injection treatment for anal outlet obstruction in patients with internal anal sphincter achalasia and Hirschsprung's disease. Pediatr Surg Int. 2009;25(10):873-876. 